\title{
Intelligent Traffic Control System Implementation for Traffic Violation Control, Congestion Control and Stolen Vehicle Detection
}

\author{
https://doi.org/10.3991/ijes.v5i2.7230 \\ Swarup Kulkarni \\ Savitribai Phule Pune University, Maharashtra, India \\ swarupkulkarni4@gmail.com \\ Dr. Roshani Ade \\ Savitribai Phule Pune University, Maharashtra, India \\ Rosh513@gmail.com
}

\begin{abstract}
Traffic is significant issue in our nation, particularly in urban ranges. Aftereffect of this, activity clog issue happens. Crisis vehicle like rescue vehicle, fire unit, squad cars confront bunches of issue to achieve their goal on account of congested driving conditions, coming about loss of human lives. To minimize this issue, we approach new idea named as "Traffic control framework for blockage control and stolen Vehicle location". In this framework activity freedom done by transforming Red flag into Green flag. We demonstrate an idea of what we call "Green wave". Alongside this, we distinguish stolen vehicle by utilizing extremely advantageous RFID innovation. In the event that stolen vehicle is been distinguished, the framework gives ready sign through ringer. Framework sends Message with the assistance of GSM to Police station. In this framework we Use diverse RFID labels for recognizing rescue vehicle and the stolen Vehicle. On the off chance that Red flag is on and IR sensor is initiated, then framework gives ringer alarm to movement police. This is novel framework which encourage great answer for comprehend traffic clog.
\end{abstract}

Keywords-ARM 7 Microcontroller, LCD Display, RFID Tag, RFID Reader, GSM, Infrared(IR) SENSOR

\section{Introduction}

Fundamental Aim to actualize this framework is to distinguish Emergency vehicle like Ambulance utilizing RFID innovation and following stolen vehicle. In customary framework, they utilize image processing to recognize crisis vehicles. Inconvenience of this framework is amid terrible climate conditions. In stormy season or in windy season, picture captured by the camera gets bended. So it's hard to recognize required vehicle and hence we actualize Reliable RFID based framework which gives continuous system correspondence regardless of the possibility that the climate condition is bad. 
India is prominent improvement nation. This paper concentrates on freedom of movement clog in urban region. Usually traffic issues occur as a result of drastically increment of vehicle in city ranges.

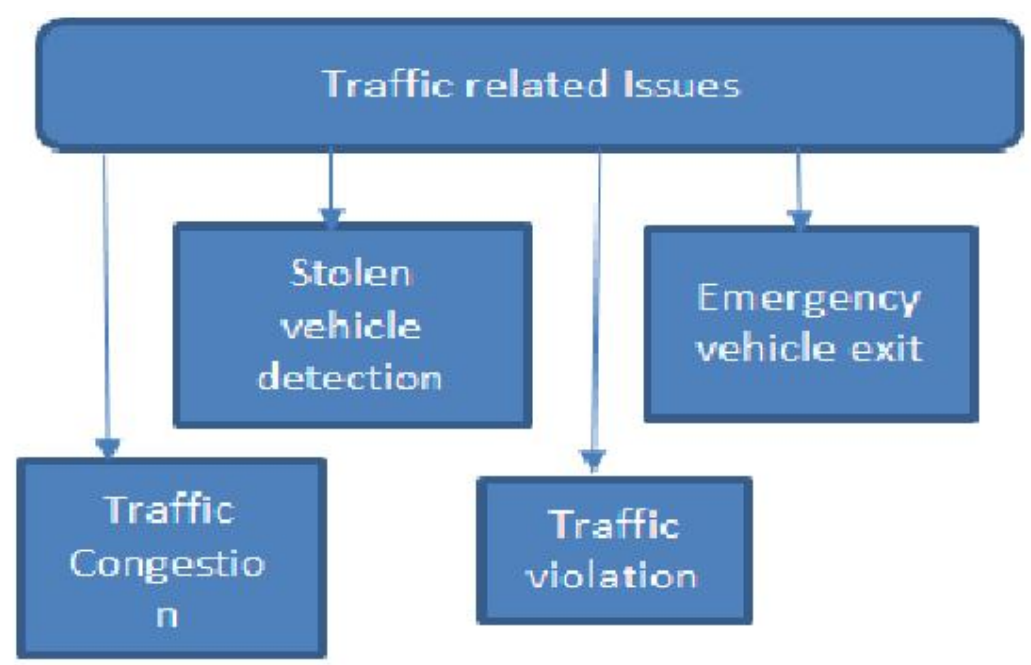

Fig. 1. Traffic Issues

Advance features of RFID, GSM give practical answer for past framework. For that reason, we will use RFID Tag to recognize stolen vehicle, emergency vehicle. RFID is a unique innovation that utilizes radio recurrence to convey data between the RFID tag and RFID reader. RFID tag is accessible in two sort Active RFID tag and Passive RFID tag. We are utilizing Passive RFID tag.

Inactive label implies no need of outside power. So battery upkeep issue is killed. Dynamic RFID label needs Supply to enact. We utilize Passive reader. GSM is a creative modem having SIM card opening and this module is actuated over a membership to portable administrator, much the same as cell phone. This modem works on AT summons. We go AT summons through Microcontroller. IR sensor emits infrared waves. An IR sensor can gauge the heat of an object and also distinguishes the movement. These types of sensors measure just the infrared radiation, instead of transmitting it. These are called as a detached IR sensor. In this module, basic IR drove and IR photodiode is accessible. At the point when any impediment interacts with it, it gives high flag to microcontroller. LCD implies Liquid Crystal Display. We utilize $16 * 2$ shows in our framework. LCD has numerous applications like PC screens, TV and so on. We utilize two LCD in shading green and red to tell activity light. At the point when rescue vehicle is identified around, then red light is supplanted with green, which implies to framework give green activity flag.

Buzzer is an electronic device that produces sound. Piezzo signal depends on the reverse rule of piezoelectricity. It produces power when mechanical weight is connected to specific materials and the other way around is likewise valid. Such materials are known as piezoelectric material. 


\section{Review of Literature}

In the paper "Intelligent traffic control system", the Intelligent Traffic Control and Management System utilizes shrewd parts like RFID, IR sensors, microcontroller, and so on[1]. It likewise comprises of modules for

- Allowing section of organized vehicles viz Ambulance, VIP, and so forth

- Enabling clients to track their stolen or lost vehicles.

- Help individuals to get data about the activity traffic in particular zone

- Avoiding debasement via computerized fine reasoning

- RFID labels are utilized for novel ID of vehicles and IR sensors are utilized to get the vehicle tally.

In the paper "Implementing intelligent traffic control system for congestion control, ambulance clearance, and stolen vehicle detection" it is around a clever movement control framework to pass crisis vehicles easily[2]. Every individual vehicle is outfitted with exceptional radio recurrence ID (RFID) tag (set at a key area), which makes it difficult to evacuate or wreck. We utilize RFID per user, NSK EDK-125TTL, and PIC16F877A framework on-chip to peruse the RFID labels joined to the vehicle. It checks number of vehicles that passes on a specific way amid a predefined span. It additionally decides the system clog, and henceforth the green light term for that way. On the off chance that the RFID-label read has a place with the stolen vehicle, then a message is sent utilizing GSM SIM300 to the police control room. What's more, when a rescue vehicle is moving toward the intersection, it will impart to the activity controller in the intersection to turn $\mathrm{ON}$ the green light. This module utilizes ZigBee modules on CC2500 and PIC16F877A framework on-chip for remote interchanges between the rescue vehicle and movement controller. The model was tried under various blends of contributions to our remote correspondence research facility and exploratory results were found not surprisingly.

Paper "A novel approach to implement green wave system and detection of stolen vehicles" explains about today's reality in which car influxes amid surge hours is one of the real concerns[3]. Amid surge hours, crisis vehicles like Ambulances, Police autos and Fire Brigade trucks stall out in jams. Because of this, these crisis vehicles are not ready to achieve their goals in time, coming about into lost human lives. We have built up a framework which is utilized to give leeway to any crisis vehicle by turning all the red lights to green on the way of the crisis vehicle, henceforth giving a total green wave to the craved vehicle.

'Green wave' is the synchronization of the green period of movement signs. With a 'green wave' setup, a vehicle going through a green signal will keep on receiving green signals as it goes not far off. Around the globe, green waves are utilized to awesome impact. Frequently criminal or psychological militant vehicles must be recognized. Notwithstanding the green wave way, the framework will track a stolen vehicle when it goes through a movement light [4] .The paper "Smart traffic control system for emergency vehicle clearance" says that the Traffic clog is real issue in urban communities of creating nations like India. The point of this venture is to pass 
crisis vehicles like VIP Vehicles, ambulances, fire trucks to their goals at the most punctual by utilizing insightful activity control framework. Then we can distinguish stolen vehicles by utilizing this control framework. Here we have executed by reaching out to every one of the streets in a multi-street intersection. In this framework every individual vehicle is furnished with extraordinary radio frequency identification (RFID) tag (put at a vital area), which makes it difficult to evacuate or obliterate. We utilize RFID per user, NSK EDK-125-TTL, LPC2148 a framework on-chip to peruse the RFID labels joined to the vehicle. It checks number of vehicles that passes on a specific way amid a predefined length. Relies on the density of vehicles on the specific intersection, the movement signs will fluctuate. In this, RFID tags are the vehicles and the Reader which is at Traffic control room, gets the information when the vehicle is stolen and the GSM will send the Message to the concern authority so that, the ready area is dynamic. We can track the stolen vehicle by utilizing this framework.

The paper "Smart driving of a vehicle using model predictive control for improving traffic flow" shows that traffic management on street systems is a rising look into field in control designing because of the solid request to reduce activity blockage in urban ranges[5]. Connection among vehicles every now and again causes clog and also bottlenecks in street limit. In thick activity, influxes of movement thickness engendering reverse as drivers attempt to be careful separations through regular increasing speed and deceleration. This paper exhibits a vehicle driving framework in a model prescient control structure that successfully enhances activity stream. The vehicle driving framework controls safe inter vehicle movement under the limited driving torque condition by foreseeing the previous activity. It likewise concentrates on easing the impact of breaking on the vehicles that take after, which helps sticking waves weaken to in the movement.

\section{$3 \quad$ Existing System}

- They are using traffic system which is not capable to control the traffic because traffic is major issue in our Country, especially in urban areas.

- This traffic congestion results in many challenges.

- They are using the systems that do not identify the stolen vehicles.

- Emergency vehicle like ambulance, fire brigade, police cars face lots of problem to reach their destination because of traffic Jam, resulting loss of human lives.

\section{$4 \quad$ Proposed System}

Proposed system has following important concepts:

- In this system, traffic clearance is done by turning Red signal into Green signal. We prove concept of "Green wave". Along with this we identify stolen vehicle by using very convenient RFID technology.

- If stolen vehicle detected, system gives alert indication through buzzer.

- System is provided by sending the Message with the help of GSM to Police station. 
- In this system we Use Different RFID tags for identify ambulance, stolen vehicle.

- If Red signal is on, and IR sensor activated system give buzzer alert to Traffic police man.

- This is unique system which facilitate good solution to solve traffic congestion

\subsection{Implementation Status}

- In this paper we use latest technology to identify the stolen vehicle \& theft i.e. RFID.

- If theft is detected then the alert message is sent to the police, for sending the message we are using GSM technology.

\section{System Architecture}

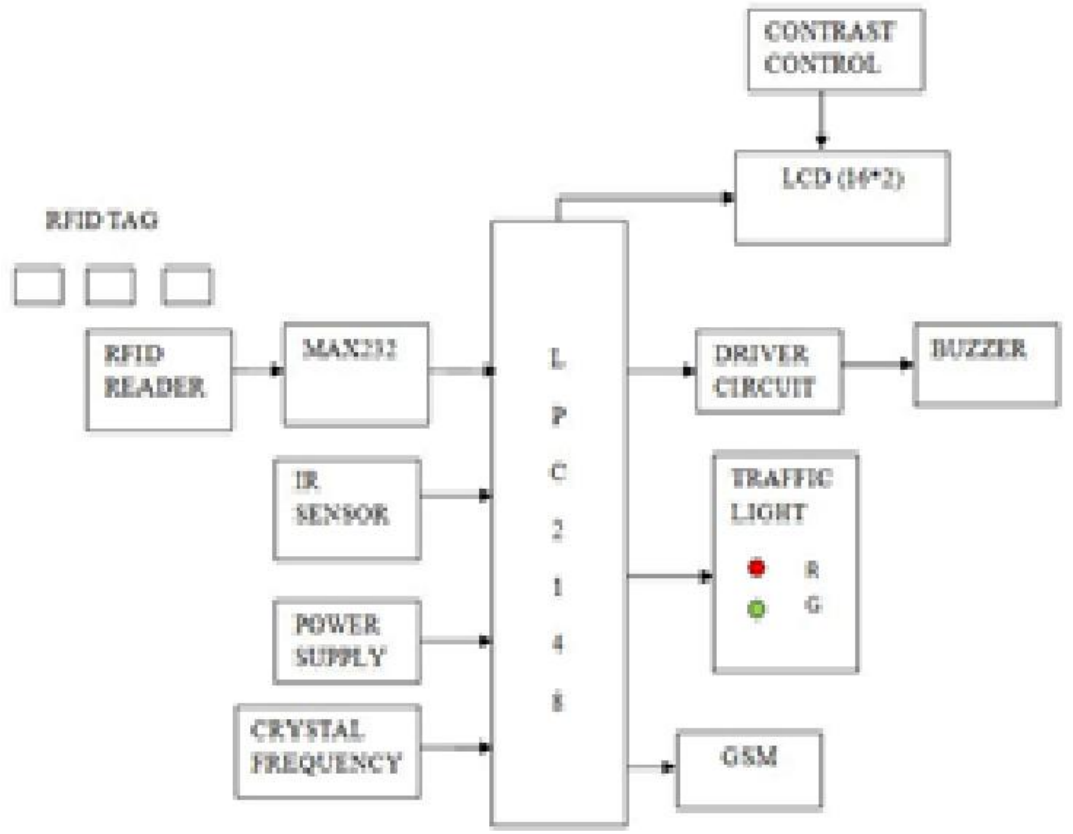

Fig. 2. Block Diagram of Proposed System

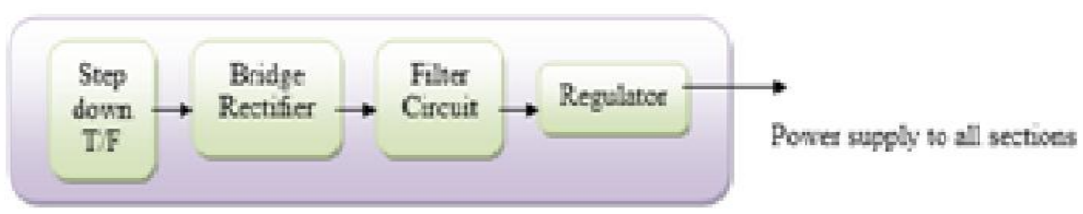

Fig. 3. Block Diagram of Power Supply 


\subsection{System Description}

In this project the prerequisite is that each vehicle is inserted with RFID tag. RFID reader peruses the vehicle tag and recognize vehicle for activity administration. If first label discharges to RFID reader, framework comprehends vehicle is approved individual. If second tag discharges, framework Identifies it is an emergency vehicle, in the meantime red flag practices environmental safety and alphanumeric display shows string "ambulance detected". If third tag is recognized, implies vehicle is stolen and the Buzzer beeps. After that an SMS as "Stolen Vehicle detected" is sent to police station.

If a vehicle tries to jump the signal, the IR sensor sends the signal to the processor, which in turn displays "Penalty" on the display board and eventually a predefined amount is deducted from his bank balance.

This project utilizes directed $5 \mathrm{v}, 750 \mathrm{~mA}$ power supply. 7805, a three terminal voltage controller is employed for voltage direction. Connect sort full wave rectifier is utilized to correct the AC yield of secondary of 230/12v stage down electrical device.

\section{Hardware Description}

\subsection{ARM 7 Microcontrollers}

The ARM7TDMI-S is a universally useful 32-bit chip, which offers elite and low power utilization. The ARM design depends on Reduced Instruction Set computer (RISC) standards, and the direction set and related disentangle element are abundant more easy than those of miniaturized scale changed complicated Instruction Set Computers (CISC). This effortlessness brings concerning a high guideline turnout and nice continuous interfere with reaction from to a small degree and savvy processor center. Pipeline strategies area unit utilized with the goal that all elements of the getting ready and memory frameworks will work perpetually. Regularly, while one direction is being dead, its successor is being decoded, and a third guideline is being brought from memory.

Details: There are 16-bit/32-bit ARM7TDMI-S microcontroller in a minor LQFP64 bundle and eight $128 \mathrm{~K} \times 8$ SRAM chips forming a $256 \mathrm{~K} \times 32$ memory unit with 128-piece wide interface/quickening agent empowers rapid sixty rate operation. The inserted ICE RT and embedded trace interfaces offer in progress work with the On-chip Real Monitor programming and quick following of guideline execution. It also has a USB 2.0 full-speed agreeable gismo controller with two computer memory unit of terminus RAM.

\subsection{IR Sensor}

An Infrared (IR) sensor is utilized to identify obstructions before the robot or to separate between hues relying upon the design of the sensor. An IR sensor comprises of an emitter, identifier and related hardware. The circuit required to make an IR 
sensor comprises of two sections; the emitter circuit and the beneficiary circuit. An infrared sensor (IR sensor) is an electronic sensor that measures infrared (IR) light emanating from items in its field of view. They are frequently utilized as a part of PIR-based movement detectors.IR Sensors work by utilizing a particular light sensor to distinguish a select light wavelength in the Infra-Red (IR) range.

Details:

- Operating voltage $-+5 \mathrm{v}$ DC controlled

- Obstacle discovery:- Indicated by dynamic high yield Logic yield:- 1 or 0.

- Sensitivity:- up to $30 \mathrm{~cm}$ flexible.

- LCD $16 * 2$ specification

- LCD stands for Liquid Crystal Display

\section{Particulars:}

- Show: - 16 Char* 2 Lines

- Controller:-LSI HD44780 IN BUILT Control Supply: - + 5v Dc

- Show Color:- Gray Weight: - 35g

\subsection{RFID Tag}

A radio-frequency identification framework utilizes labels, or names joined to the items to be recognized. Two-way radio transmitter-collectors called investigators or readers send a flag to the tag and read its reaction. RFID labels can be passive, active or battery-assisted passive. An active tag has an on-board battery and intermittently transmits its ID flag. A battery-assisted passive (BAP) has a little battery on board and is actuated when within the sight of a RFID reader. A latent tag is less expensive and littler in light of the fact that it has no battery; rather, the label utilizes the radio vitality transmitted by the reader. Be that as it may, to work an uninvolved label, it must be lit up with a power level about a thousand circumstances more grounded than for flag transmission. That has any kind of effect in obstruction and in presentation to radiation.

\subsection{RFID Reader}

RFID frameworks can be arranged by the sort of tag and reader. A Passive Reader Active Tag (PRAT) framework has an inactive reader which just gets radio signs from dynamic labels (battery worked, transmit as it were). The gathering scope of a PRAT framework reader can be balanced from 12,000 feet $(0600 \mathrm{~m})$, permitting adaptability in applications, for example, resource security and supervision.

An Active Reader Passive Tag (ARPT) framework has a dynamic reader, which transmits questioner signals furthermore gets validation answers from aloof labels. An Active Reader Active Tag (ARAT) framework utilizes dynamic labels awoken with a cross examiner motion from the dynamic reader. A variety of this framework could likewise utilize a Battery-Assisted Passive (BAP) label which acts like a latent tag yet has a little battery to control the label's arrival reporting signal. Repaired readers are 
set to make a particular cross examination zone which can be firmly controlled. This permits an exceptionally characterized perusing range for when labels go all through the cross examination zone. Versatile readers might be hand-held or mounted on trucks or vehicles.

Specifications:

- Operating Voltage - 5v Current- $<50 \mathrm{~mA}$

- Read separate $10 \mathrm{~cm}$

- Operating recurrence $125 \mathrm{khz}$

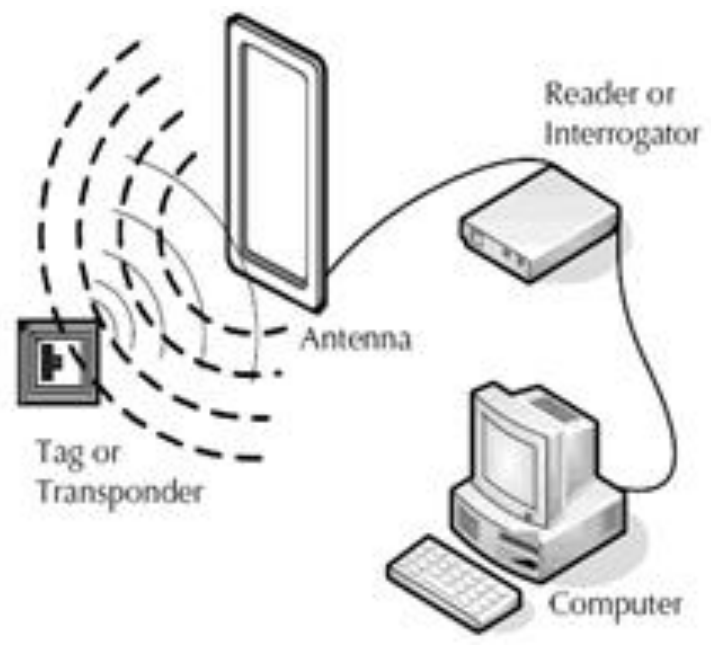

Fig. 4. RFID

Technical characteristics of active and passive RFID: Despite the fact that they both fall under the "RFID" moniker and are regularly examined conversely, Active RFID and Passive RFID are in a general sense distinctive advancements. While both utilize radio recurrence vitality to impart between a tag and a reader, the technique for driving the labels is distinctive. Active RFID utilizes an inside power source (battery) inside the tag to ceaselessly control the tag and its RF correspondence hardware, though Passive RFID depends on RF vitality exchanged from the reader to the tag to control the tag. While this refinement may appear to be minor at first glance, its effect on the usefulness of the framework is huge.

Aloof RFID either Reflects vitality from the reader or Ingests and incidentally stores a little measure of vitality from the reader's tag to produce its own particular speedy reaction. In either case, passive RFID operation requires exceptionally solid signs from the reader, and the flag quality came back from the tag is compelled to low levels by the constrained vitality. Then again, Active RFID permits low-level signs to be gotten by the tag (in light of the fact that the reader does not have to control the tag), and the tag can create abnormal state motions back to the reader, driven from its inward power source. Also, the Active RFID tag is ceaselessly controlled, whether in the reader field or not. As talked about in the following segment, these distinctions 
affect correspondence go, multi-label gathering capacity, capacity to include sensors and information logging, and numerous other useful parameters.

Active RFID and passive RFID advances, while regularly considered and assessed together. They are in a general sense have unique abilities. By and large, neither one of the technology's gives a total answer for inventory network resource administration applications. Or maybe, the best and finish inventory network arrangements influence the upsides of every innovation and consolidate their utilization in reciprocal ways. This requirement for both advancements must be considered by RFID models activities to adequately meet the prerequisites of the client group.

\subsection{Buzzer}

A buzzer or beeper is a sound flagging gadget, which might be mechanical, electromechanical, or piezoelectric. Regular employments of ringers and beepers incorporate alert gadgets, clocks, and affirmation of client info, for example, a mouse snap or keystroke. Piezo buzzer is an electronic gadget usually used to create sound. Light weight, basic development and low value make it usable in different applications like auto/truck switching marker, PCs, call chimes and so on. Piezo bell depends on the converse guideline of piezo power found in 1880 by Jacques and Pierre Curie. It is the wonders of creating power when mechanical weight is connected to specific materials and the other way around is additionally valid. Such materials are called piezo electric materials.

Piezo electric materials are either actually accessible or synthetic. Piezo clay is class of synthetic material, which postures piezo electric impact and is generally used to make plate, the heart of piezo signal. At the point when subjected to a rotating electric field they extend or pack, as per the recurrence of the flag in this way creating sound.

\section{Specification:}

- Voltage : 2 - 5VDC

- Maximum current : 30mA/5VDC Decibel : $85 \mathrm{db} / 10 \mathrm{~cm}$

- Resonant frequency : $2500 \mathrm{~Hz}(+/-300 \mathrm{HZ})$ Operating Temperature : -20 to $70 \mathrm{C}$

\section{Points of interest of RFID:}

- The development of stock can be followed

- Products can get easily and dispatched earlier.

- Simplicity in the item request.

- Customers can spare time.

- Out of stock circumstances can be stayed away from customers.

- The correct items are accessible at the correct stores at the ideal time.

Components of RFID labels: RFID labels characterization, with or without an electronic chip. A first characterization for RFID labels depends on the nearness or nonappearance of an electronic chip. RFID SAW labels (Surface Acoustic Wave) don't have coordinated circuits. Today they just speak to a little part of the market (a 
couple percent). They are perused just transponders and do exclude installed vitality. They are likewise called RF standardized identification.

1 bit RFID labels are in active frameworks with capacitive diodes, called as " 1 bit transponders." This bit shows if the tag is available of not in the cross examiner's field of activity. They are broadly utilized as hostile to burglary framework. RFID labels with coordinated circuits are the most utilized frameworks on the real market. They are made out of a radio wire and an incorporated circuit.

\subsection{MAX232}

The is an incorporated circuit initially made in 1987 by Maxim Integrated Products that proselytes signals from a TIA-232 (RS-232) serial port to signals reasonable for use in TTL good computerized rationale circuits. The MAX232 is a double driver/recipient and ordinarily changes over the RX, TX, CTS and RTS signals. The MAX232 is an incorporated circuit initially made in 1987 by Maxim Integrated Products that proselytes signals from a TIA-232 (RS-232) serial port to signals appropriate for use in TTL good computerized rationale circuits. The MAX232 is a double driver/beneficiary and regularly changes over the RX, TX, CTS and RTS signals. The drivers give TIA-232 voltage level yields (approx. 7.5 volts) from a solitary five volt supply by means of on-chip charge pumps and outer capacitors. This makes it valuable for executing TIA-232 in gadgets that generally needn't bother with whatever other voltages.

\section{$7 \quad$ Software Requirement}

- Programming languages: Embedded

- Compilers: Keil 4.0uv

- Dumping software: using micro controller flash magic software we are dumping our hex code into micro controller.

\subsection{System Performance}

In this system we use RFID to identify the authenticated Vehicle owner \& stolen vehicle. We can easily find out the theft it will more helpful to the police because when the theft is detected buzzer will give beep at that time GSM technology will send Message to the police and GPS will send the exact location of the theft.

This paper is giving important performance to identify the emergency vehicles like Ambulance and fire vehicle. This system will use full in emergency.

\section{Mathematical Module}

Let $\mathrm{W}$ be the whole system which consists Input $=\{R\}$. 
Let $\mathrm{R}$ is the set of number of RFID user

$\mathrm{R}=\left\{r_{1}, r_{2}, \ldots ., r_{n}\right\}$

Let I be the IR sensor check for vehicle not follow traffic rule.

$\mathrm{I}=\left\{i_{1}, i_{2}, \ldots ., i_{n}\right\}$

\subsection{1 Verification of RFID tag}

Step 1:- When the vehicle arrives near the signal pole, accept its RFID.

Step 2:- If the vehicle is authorized, no action taken

Step 3:-If ambulance detected, instantly the traffic signal gets changed to green.

Step 4:-If stolen vehicle detected, instantly the time buzzer gets activated for alert.

\subsection{Check for IR sensor}

Step 1:- If any vehicle violates the signal, its being detected through the IR sensor Step 2:- At that time buzzer get activated.

Step 3:- Display on LCD as "penalty".

\subsection{Efficiency Calculations}

- Microcontroller $-5 \mathrm{v}$

- Transformer 230 to $12 \mathrm{v}$

- Filter capacitor - $1000 \mathrm{uf}, 25 \mathrm{v}$

- Voltage regulator - 7805

- RFID Power Consumption - 3.3V, 13-26mA

- GSM power consumption: $3.3 \mathrm{v}, 1.5 \mathrm{~mA}$ 


\subsection{System Design}

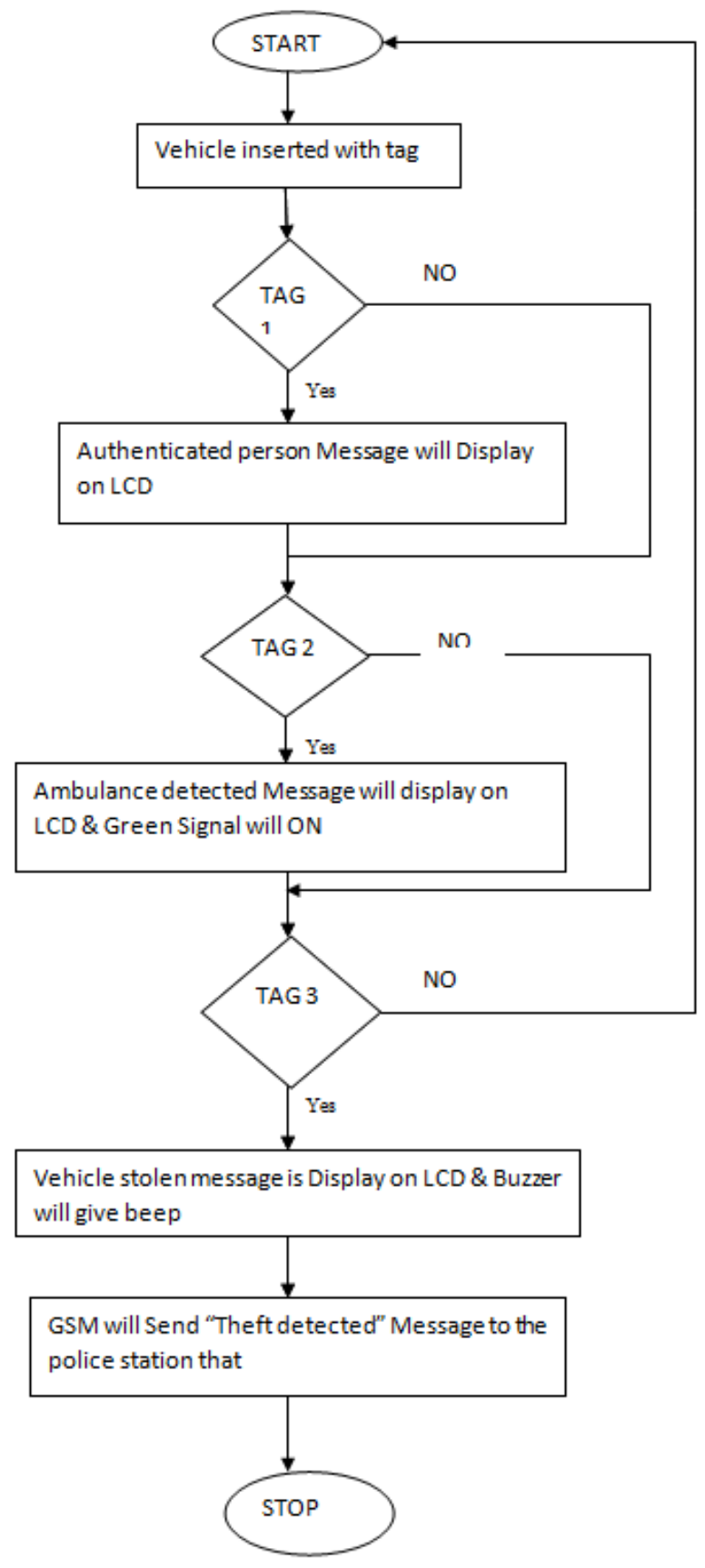

Fig. 5. System Design 


\section{Result of the System}

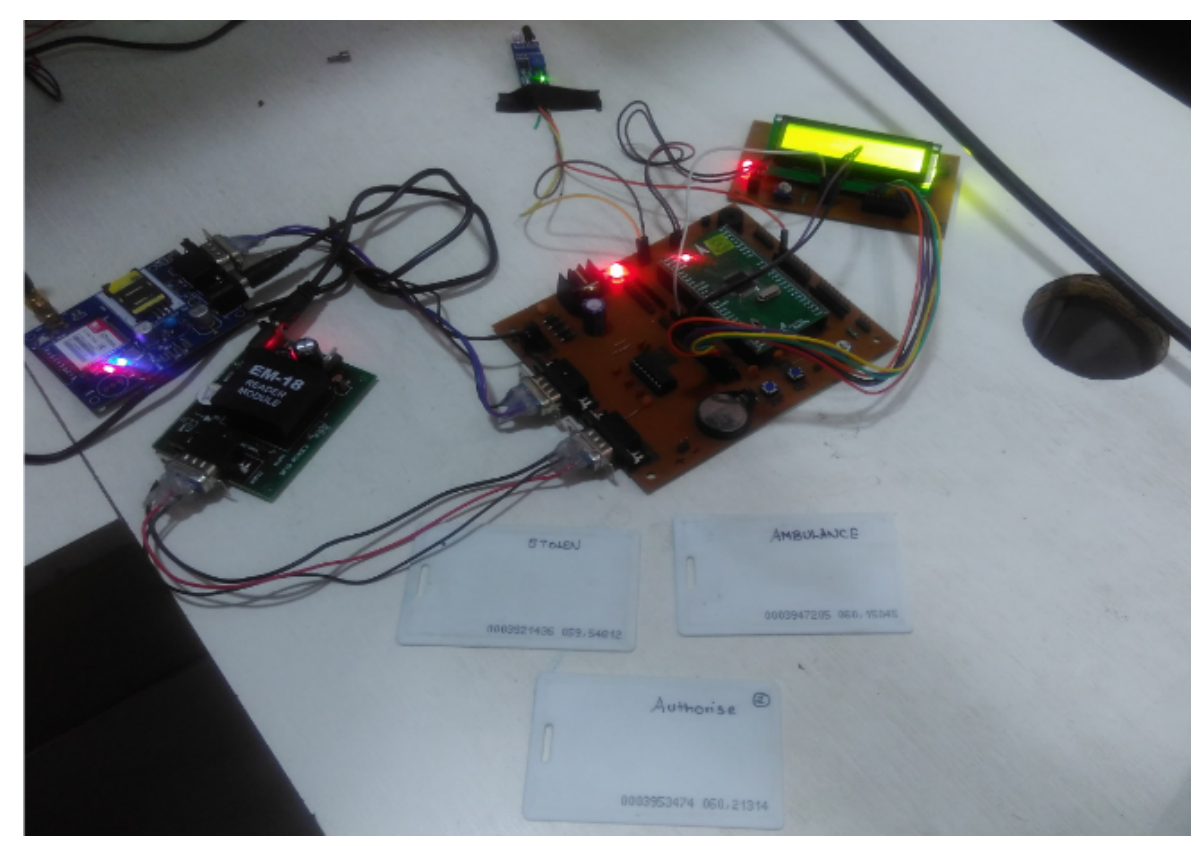

Fig. 6. Actual Circuit Boards

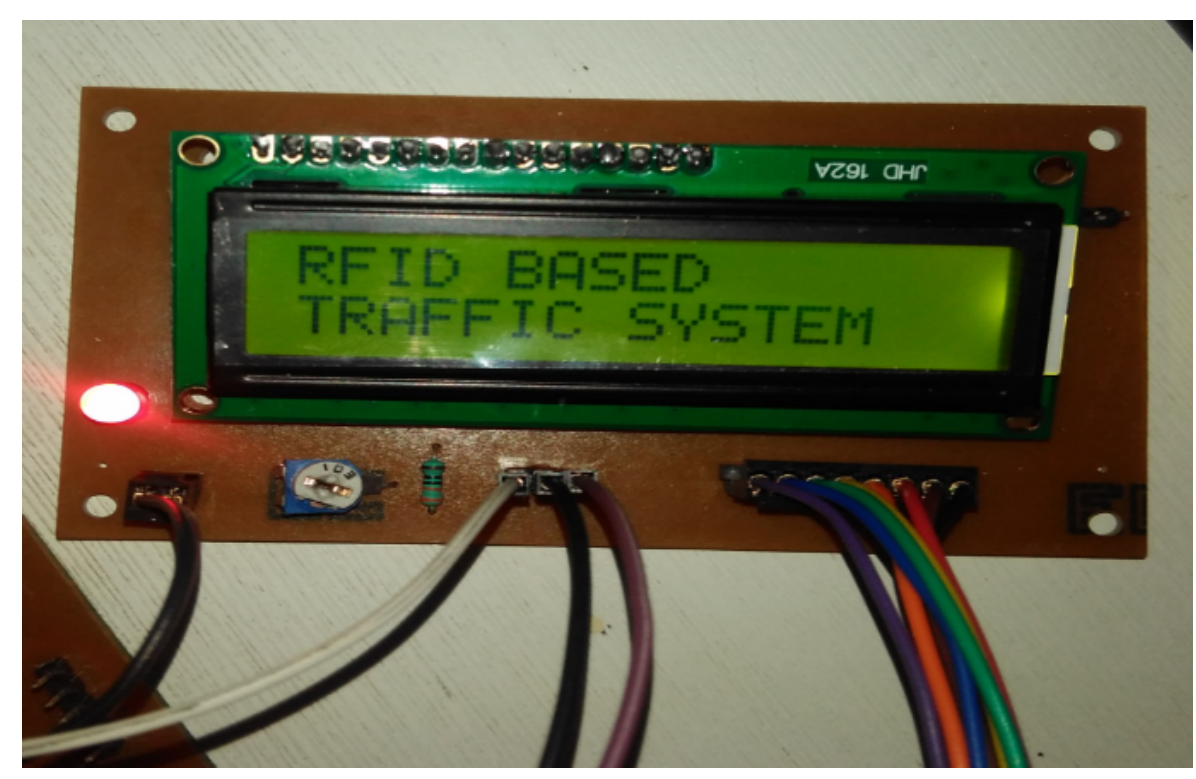

Fig. 7. RFID Based Traffic System Message 


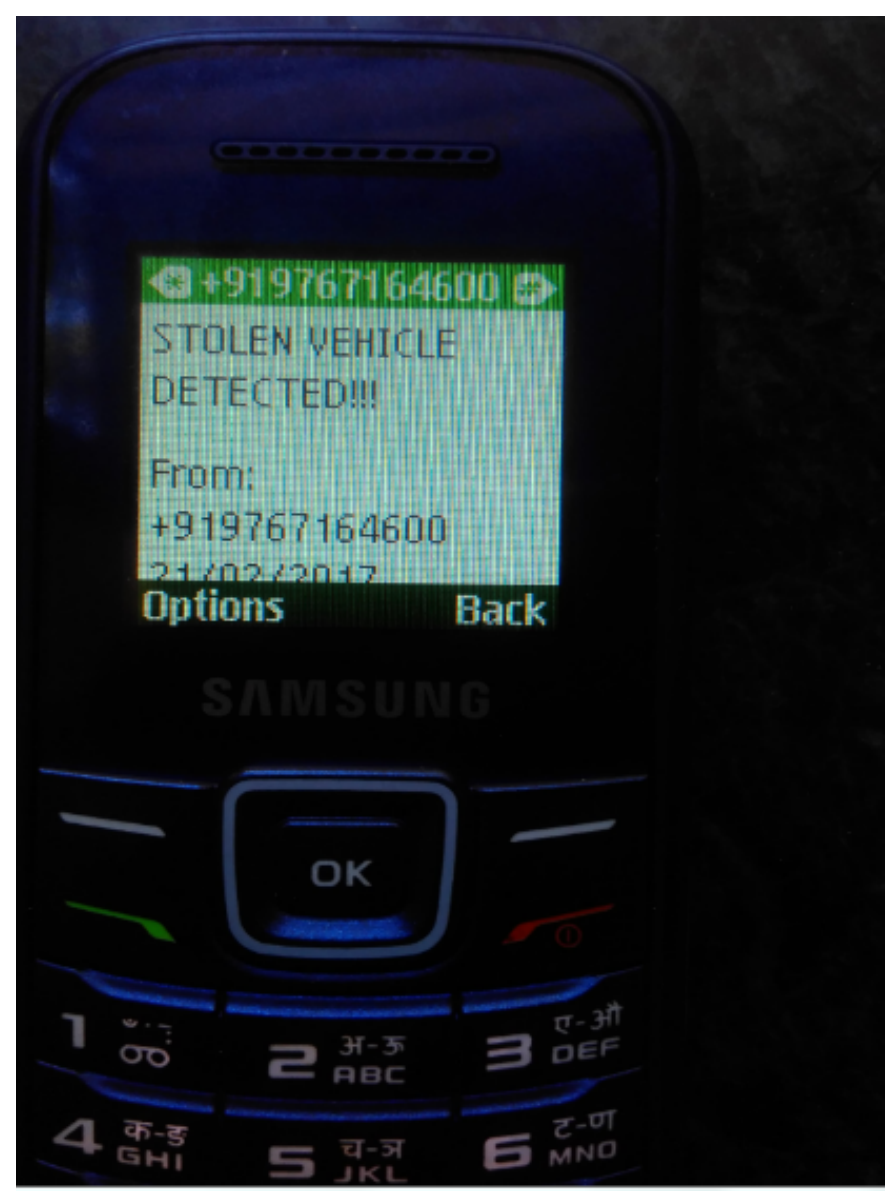

Fig. 8. Actual Result

\section{Conclusion}

As the whole system is automated, it requires less human mediation. With stolen vehicle discovery conceivable intersections ready message notice sending done alongside ringer sign. Emergency vehicles like ambulance need to achieve their goals at the most punctual. In the event that they invest a considerable measure of energy in congested roads. With crisis vehicle freedom, the activity flag swings to green and the length of the crisis vehicle is holding up in the movement intersection. The signal swings to red, simply after the crisis vehicle goes through. As of now, it is actualized framework by thinking of one as street of the activity intersection. In future for improve framework we Add ZIGBEE Module to upgrade framework As ZIGBEE extremely helpful in remote sensor arrange. We can utilize Road Divider idea. At the point when activity thickness High at one side Divider will move to another side for leeway of movement. 


\section{Acknowledgment}

I wish to express my true on account of my guide, for her significant direction. Without her direction this work would not have been conceivable. I am appreciative for her helping nature which helped me to finish this work on time. I might likewise want to express my appreciation to, Principal and Head of Computer Department for giving such an open door. I additionally express my appreciation for all the staff of Computer Engineering Dept. for their support. I additionally express my gratitude to my parents and my companions for their important support.

\section{References}

[1] Prof. Mrs Vidya Patil, "Intelligent Traffic Control System" International Journal of Engineering Technology, Management and Applied Sciences, February 2016.

[2] Sundar, Rajeshwari, Santhoshs Hebbar, and Varaprasad Golla, "Implementing intelligent traffic control system for congestion control, ambulance clearance, and stolen vehicle detection" IEEE Sensors Journal 15.2 (2015): 1109-1113. https://doi.org/10.1109/JSEN. 2014.2360288

[3] Mittal, Ayush Kr, and Deepika Bhandari, "A novel approach to implement green wave system and detection of stolen vehicles", Advance Computing Conference (IACC), 2013 IEEE 3rd International, 2013.

[4] Veera Venkatesh, Nazneen Syed, "Smart Traffic Control System for Emergency Vehicle Clearance", International Journal of Innovative Research in Computer and Communication Engineering, Vol. 3, Issue 8, August 2015.

[5] Abdus Samad Kamal Md, Jun-ichi Imura, Tomohisa Hayakawa, Akira Ohata, and Kazuyuki Aihara (2014), "Smart Driving of a Vehicle Using Model Predictive Control for Improving Traffic Flow"e, IEEE Trans. Intell. Transp.Syst, vol. 15, no. 2

[6] Arunmozhi.P, Joseph William.P (2012), “Automatic Ambulance Rescue System Using Shortest Path Finding Algorithm", International Journal of Science and Research, vol.3, Issue 5.

[7] Andrew Phan and Frank P. Ferrie (2011), "Interpolating Sparse GPS Measurements Via Relaxation Labeling and Belief Propagation for the Redeployment of Ambulances", IEEE Trans. Intell. Transp. Syst, vol. 12, no. 4. https://doi.org/10.1109/TITS.2011.2165281

[8] Akyildiz.I.F, W. Su, Y. Sankarasubramaniam, and E. Cayirci(2002), “A survey on sensor networks", IEEE Communications Magazine, Vol. 40, pp. 102- 114. https://doi.org/10.1109/MCOM.2002.1024422

\section{Authors}

Swarup Kulkarni is pursuing Master of Engineering degree from Dr. D. Y. Patil School of Engineering and Technology, Pune of Savitribai Phule Pune University. He has received B. E. degree in Information technology.

Dr. Roshani Ade is working as a Professor in Dr. D. Y. Patil School of Engineering and Technology, Pune of Savitribai Phule Pune University, India.

Article submitted 30 May 2017. Published as resubmitted by the authors 03 July 2017 